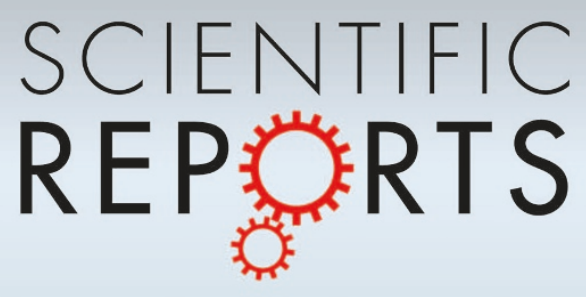

OPEN

SUBJECT AREAS:

ELECTRONIC PROPERTIES

AND MATERIALS

SURFACES, INTERFACES AND

THIN FILMS

Received

21 July 2014

Accepted

9 October 2014

Published

29 October 2014

Correspondence and requests for materials should be addressed to S.M. (smiddey@uark. edu)

\section{Polarity compensation in ultra-thin films of complex oxides: The case of a perovskite nickelate}

\author{
S. Middey' , P. Rivero', D. Meyers' ', M. Kareev' ', X. Liu', Y. Cao', J. W. Freeland², S. Barraza-Lopez \\ \& J. Chakhalian'
}

'Department of Physics, University of Arkansas, Fayetteville, Arkansas 72701, USA, ${ }^{2}$ Advanced Photon Source, Argonne National Laboratory, Argonne, Illinois 60439, USA.

We address the fundamental issue of growth of perovskite ultra-thin films under the condition of a strong polar mismatch at the heterointerface exemplified by the growth of a correlated metal $\mathrm{LaNiO}_{3}$ on the band insulator $\mathrm{SrTiO}_{3}$ along the pseudo cubic [111] direction. While in general the metallic $\mathrm{LaNiO}_{3}$ film can effectively screen this polarity mismatch, we establish that in the ultra-thin limit, films are insulating in nature and require additional chemical and structural reconstruction to compensate for such mismatch. A combination of in-situ reflection high-energy electron diffraction recorded during the growth, X-ray diffraction, and synchrotron based resonant $\mathrm{X}$-ray spectroscopy reveal the formation of a chemical phase $\mathrm{La}_{2} \mathrm{Ni}_{2} \mathrm{O}_{5}\left(\mathrm{Ni}^{2+}\right)$ for a few unit-cell thick films. First-principles layer-resolved calculations of the potential energy across the nominal $\mathrm{LaNiO}_{3} / \mathrm{SrTiO}_{3}$ interface confirm that the oxygen vacancies can efficiently reduce the electric field at the interface.

\begin{abstract}
$\Lambda$
rtificial heterostructures based on transition metal oxides lead to fascinating interfacial phenomena unfeasible in their bulk form ${ }^{1-4}$. For example, highly mobile two dimensional electron gas ${ }^{5}$ and superconductivity around $200 \mathrm{mK}^{6}$ have been observed at the interface between two wide band gap insulators $\mathrm{LaAlO}_{3}(\mathrm{LAO})$ and $\mathrm{SrTiO}_{3}(\mathrm{STO})$. Though the actual physical process responsible for this metallic behavior is still under debate, it has been unanimously concluded that the polar mismatch at the LAO/STO interface is responsible for this emergent metallic state ${ }^{7-13}$. With LAO/STO as a non-trivial example, the polar mismatch driven phenomena is now of great general interest and has been studied in a broader range of complex oxide materi$\mathrm{als}^{14-17}$. The extensive experimental work has demonstrated that the divergence of the electric field due to this interfacial polarity mismatch can be resolved in a number of ways, e.g. by electronic reconstruction ${ }^{7-9,14,15}$, by cationic inter-mixing ${ }^{10,16}$, or by oxygen vacancies ${ }^{11,12,18}$.
\end{abstract}

Furthermore, recent theoretical works have predicted a host of exotic ground states (e.g. Dirac half-semimetals, quantum-anomalous Hall-insulators, ferromagnetic nematics, time-reversal invariant $Z_{2}$ topological phases, Chern insulator, etc.) in heterostructures of transition metal oxides grown along the pseudo-cubic [111] direction ${ }^{19-25}$. To this end, the common substrate $\mathrm{SrTiO}_{3}$ (or $\mathrm{LaAlO}_{3}, \mathrm{NdGaO}_{3}, \mathrm{YAlO}_{3}, \mathrm{LaGaO}_{3}$, etc.) forms alternating $+4 \mathrm{e},-4 \mathrm{e}$ (or $+3 \mathrm{e},-3 \mathrm{e}$ ) charged planes stacked along this direction, leading to a large polarity mismatch at the interface (see Fig. 1(b)). This mismatch can have a significant effect on initial nucleation and growth ${ }^{26}$ and on the overall materials properties that are particularly sensitive for ultra-thin films. Based on this, the mechanism by which polar discontinuity is compensated in real materials is of paramount interest towards the experimental realization of new materials with exotic properties.

To address this issue, rare earth nickelate $\mathrm{LaNiO}_{3}$ (LNO) films were grown on a single crystal cubic STO substrate oriented along the strongly polar [111] direction. In bulk LNO, Ni is in the low-spin $3 d^{\top}$ configuration with an unusually high +3 oxidation state, i.e. $\left(t_{2 g}^{6}, e_{g}^{1}\right)$; the compound is metallic, and it remains paramagnetic down to the lowest temperature. Ultra-thin films of LNO grown along the moderately polar (001) direction have been extensively investigated recently ${ }^{27-33}$ largely due to the prediction of high-temperature superconductivity when heterostructured with the band insulator $\mathrm{LAO}^{34,35}$. The experimentally realized LNO films and LNO/LAO superlattices on STO (001), however, revealed the presence of an unexpected transition to the Mott insulating and antiferromagnetic ground state with decreasing LNO thickness ${ }^{28,30,32}$. Additionally, it was established ${ }^{27}$ that

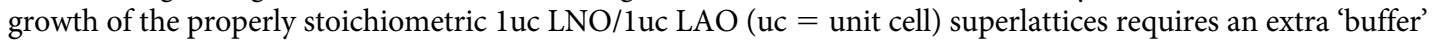




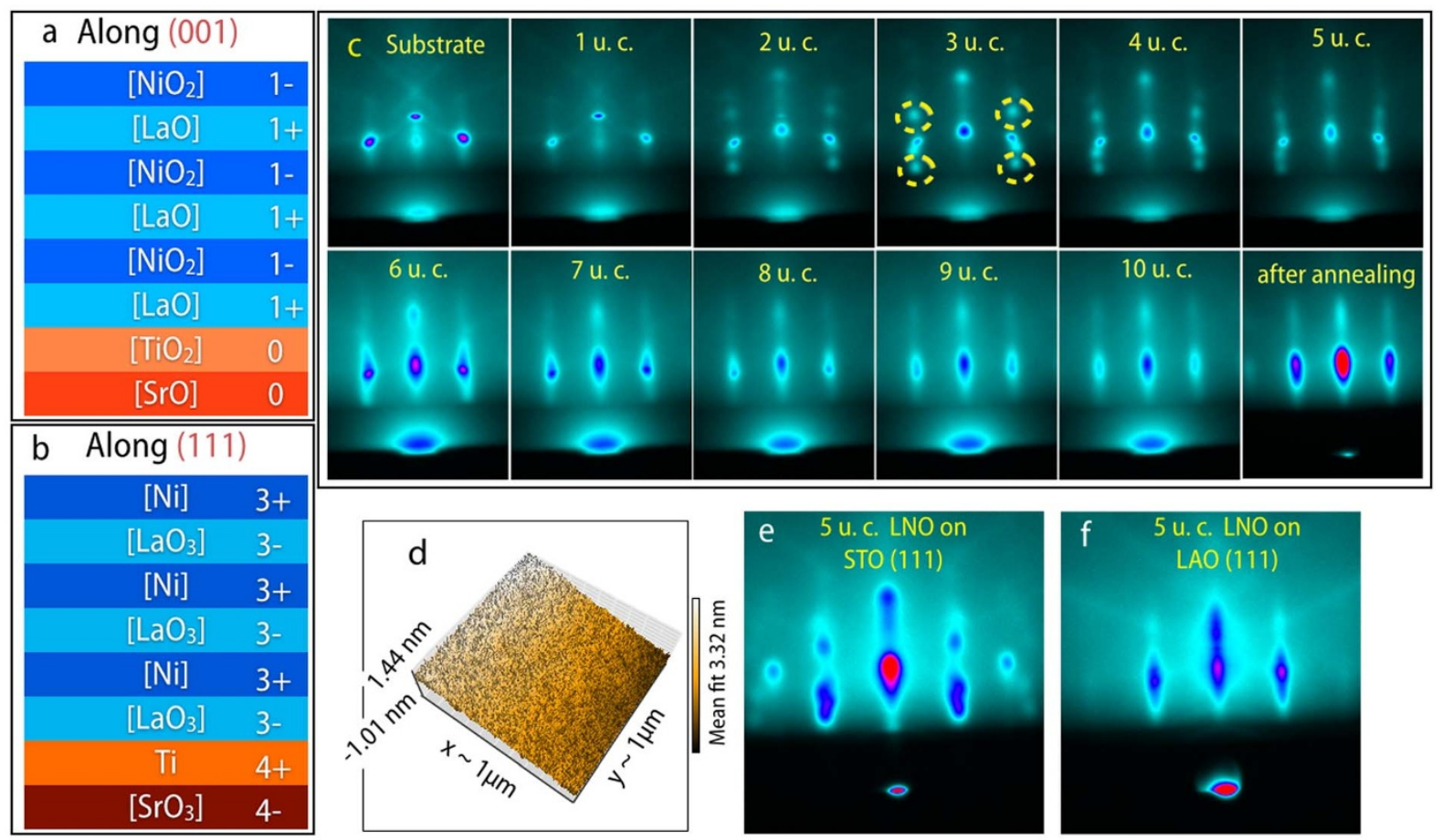

Figure $1 \mid$ Polarity mismatch and morphology during growth. (a) and (b) Schematics of polar discontinuity (in purely ionic limit and without considering any surface reconstruction). STO $\left(: \mathrm{SrO}, \mathrm{TiO}_{2}\right)$ is non-polar while $\mathrm{LNO}\left(:[\mathrm{LaO}]^{1+}\left[\mathrm{NiO}_{2}\right]^{1-}\right)$ is polar along the $(001)$ direction. Both STO (: $\left.\left[\mathrm{SrO}_{3}\right]^{4-}, \mathrm{Ti}^{4+}\right)$ and LNO (: $\left.\left[\mathrm{LaO}_{3}\right]^{3-} \mathrm{Ni}^{3+}\right)$ are strongly polar along the (111) direction. (c) Set of RHEED images recorded during growth of 10 unit cells LNO on a STO (111) substrate. RHEED patterns from bare STO (111) substrate and the film (after cooling to $300 \mathrm{~K}$ ) are also shown. The additional reflections are highlighted by the dotted ellipses in one representative image for clarity. (d) AFM image of a 10 uc LNO film. RHEED images for a 5 uc LNO film grown on (e) polar STO (111), (f) non-polar LAO (111) substrate. All RHEED images are taken along the pseudocubic [1-1 0] direction. All of the crystallographic directions and film thickness in this work are defined with respect to the pseudocubic unit cell.

monolayer of LAO to compensate for the polar mismatch, with resonant x-ray absorption on $\mathrm{Ni} L_{3,2}$-edge confirming the presence of $\mathrm{Ni}^{2+}$ in the first LNO layer ${ }^{27}$.

A direct inspection of the difference in the ionic arrangement of (001) and (111) planes shown in Fig. 1(a) and (b) implies that the expected dipole electric field mismatch is markedly more severe for ultra-thin LNO films grown along the (111) orientation and likely requires further compensation mechanisms beyond those already observed on (001) STO. Obviously, when the LNO layer becomes thicker and reestablishes its bulk-like metallicity, the polar catastrophe can be easily avoided by metallic screening, without the requirement of additional chemical, structural, or electronic reconstructions ${ }^{26}$. Here, by comprehensive experimentation combined with $a b$ initio calculations, we demonstrate how the growth of an $\mathrm{La}_{2} \mathrm{Ni}(2+)_{2} \mathrm{O}_{5}$ (LNO225) phase from the removal of oxygen from the octahedral units near the STO/LNO interface can effectively circumvent such polar catastrophe and leads to a recovery of stoichiometric layer-by-layer growth despite the highly polar interface.

Fig. 1(c) shows the set of RHEED (reflection high energy electron diffraction) patterns recorded from a bare STO (111) substrate and after each consecutive unit cell (uc) during the growth of 10 pseudocubic unit cells of LNO. First of all, the absence of any additional reflection between specular and off-specular spots in the RHEED pattern of STO (111) substrate ${ }^{36}$ (the diffraction pattern recorded under $5 \times 10^{-7}$ Torr pressure at the growth temperature has been shown in Supplemental Figure S1 for better resolution) indicates the absence of surface reconstructions at the growth conditions used in the present study. It can be clearly seen that during the initial phase of growth (from 1 uc to $5 \mathrm{uc}$ ), the RHEED pattern consists of additional Bragg reflections (highlighted by the dotted ellipses) along with the conventional specular and off-specular reflections. This observation signals the growth of additional epitaxial 3D microcrys- tallites $^{37}$, resulting in a rough, phase-impure surface. As growth continues beyond $5 \mathrm{uc}$, however, the extra spots suddenly merge and the new pattern attains the streak-like specular (and off-specular) pattern of reflections, common for electron diffraction off an atomically at $2 \mathrm{D}$ surface ${ }^{38,39}$. Direct space imaging by AFM (atomic force microscope), shown in Fig. 1(d), confirms the high morphological quality of the grown surface (average surface roughness $\sim 180 \mathrm{pm}$ ). To investigate the phases within the initial layers, growth was interrupted after reaching $5 \mathrm{uc}$, and the thin film was annealed following the same protocol used for the $10 \mathrm{uc}$ film. The retention of the additional reflection in the RHEED image even after annealing (Fig. 1(e)), and an AFM scan (not shown) with roughness $480 \mathrm{pm}$ [much higher compared to the roughness of $10 \mathrm{uc}$ film $(180 \mathrm{pm})]$ indicate that annealing can not improve the surface morphology of these thin-films. To investigate whether the observed drastic change in the growth phase is related to the polarity issue, we have performed identical growth on a LAO (111) substrate [nopolar mismatch at the LNO/LAO (111) interface]. As seen in Fig. 1(f) (see Ref. 40 for additional information) the additional reflections are entirely absent for a 5 uc LNO film grown on the LAO (111) substrate. This result (together with Fig. 1(e) for 5 uc LNO on STO (111)) suggests that the unexpected initial growth behavior on STO (111) is probably due to the polar discontinuity at the heterointerface.

To gain further insight into the structural quality and the chemical phase obtained during the initial growth sequence, we recorded Xray diffraction $2 \theta-\omega$ patterns using $\mathrm{Cu} \mathrm{K}_{\alpha}$ radiation leading to several key observations. First, as seen in Fig. 2(a), the film diffraction peak (indicated by arrows) obtained at higher $2 \theta$ than the STO (111) reflection confirms the epitaxial [111] growth under tensile strain. The relative width of this peak decreases in moving from 5 uc to $15 \mathrm{uc}$, due to the increase of film thickness. In addition, for the $5 \mathrm{uc}$ 

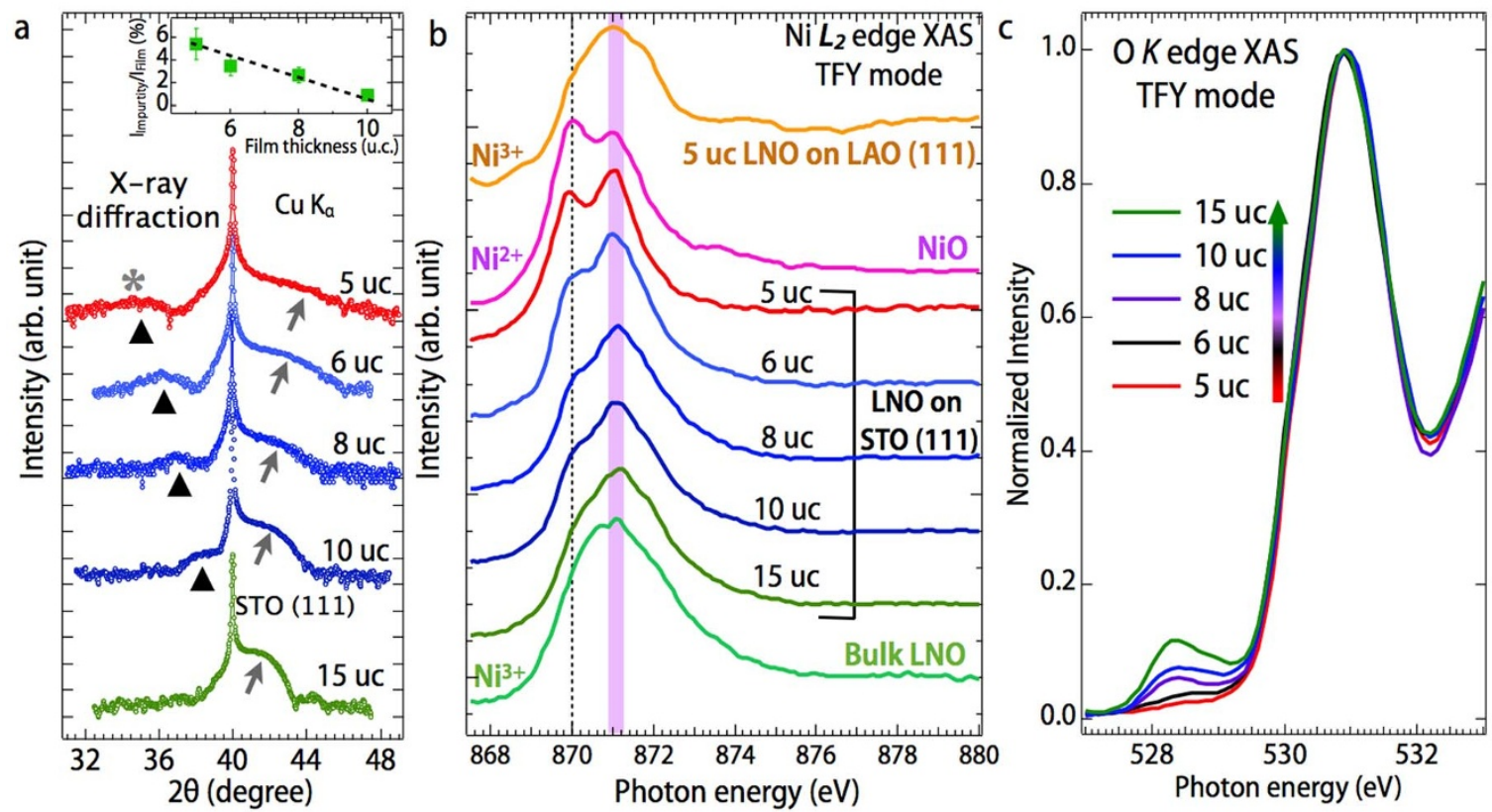

Figure $2 \mid$ X-ray diffraction and X-ray absorption spectroscopy. (a) $2 \theta-\omega$ scans for LNO films around the STO (111) reflection. The (111) film peaks and the 'impurity' peaks are indicated by arrows and solid triangles respectively. The ratio of the area under the 'impurity' peak and film peak is shown as a function of film thickness in the inset. (b) $\mathrm{Ni} L_{2}$-edge and (c) $\mathrm{O} K$-edge XAS spectra from different samples recorded in TFY mode at $300 \mathrm{~K}$. The peak at $531 \mathrm{eV}$ is due to the STO substrate.

film, an additional broad peak marked by the * has been observed around $2 \theta=35^{\circ}$ (see Fig. 2(a)). The presence of oxygen vacancies, discussed latter with absorption data, further highlights that this additional peak in XRD must originate from some oxygen deficient perovskites with formulae $\mathrm{La}_{n} \mathrm{Ni}_{n} \mathrm{O}_{3 n-1}$. This additional phase present in the $5 \mathrm{uc}$ film has a set of parallel planes with a separation of $d$ $=2.59 \AA$ which is very close to $d$ of (202) planes for the monoclinic $\mathrm{La}_{2} \mathrm{Ni}_{2} \mathrm{O}_{5}(d=2.609 \AA)^{41}$ and also for (011) plane of tetragonal $\mathrm{LaNiO}_{2}(d=2.57 \AA)^{42}$. The presence of large number of $\mathrm{Ni}$ in the +2 oxidation state there (discussed later with Fig. 2(b)) indeed confirms the formation of $\mathrm{La}_{2} \mathrm{Ni}_{2} \mathrm{O}_{5}$ and such a small deviation of the inter-plane separation from the corresponding value of bulk $\mathrm{La}_{2} \mathrm{Ni}_{2} \mathrm{O}_{5}$ likely arises from the substrate-induced strain. The origin of the gradual shifting of this peak toward higher angles as the film thickness increases above 5 uc and its absence in the 15 uc film will be discussed latter. As x-rays can penetrate deeper compared with the electron beam, the interfacial additional phase can be observed in 10 uc films, while RHEED, being a surface sensitive tool, shows a flat 2D surface (Fig. 1(c)).

To corroborate structural information from XRD and RHEED with the knowledge of the electronic structure and charge state of $\mathrm{Ni}$, we performed resonant X-ray absorption measurements on $\mathrm{Ni}$ $L_{3,2}$-edge and oxygen $K$-edge in the bulk-sensitive total fluroscence yield (TFY) mode. From the chemistry perspective, the presence of oxygen vacancies should alter the $\mathrm{Ni}^{+3}$ charge state. Fig. 2(b) shows the results of the experiment. Due to the strong overlap of the La $M_{4}$ edge with the $\mathrm{Ni} L_{3}$-edge (Supplemental Figure S5), only Ni $L_{2}$-edge spectra are used for the analysis, along with the reference spectra from the bulk $\mathrm{Ni}(2+) \mathrm{O}$ and $\mathrm{LaNi}(3+) \mathrm{O}_{3}$ compounds. A comparison of the line-shape and the $L_{2}$ energy position (i.e. chemical shift) for the $5 \mathrm{uc}$ film to the well known $\mathrm{Ni}^{2+}$ material $\mathrm{NiO}$ unequivocally indicates that $\mathrm{Ni}$ ions are indeed in the +2 oxidation state, thus providing a direct spectroscopic signature for the existence of the $\mathrm{La}_{2} \mathrm{Ni}_{2} \mathrm{O}_{5}$ phase as elucidated from the XRD data. As seen in Fig. 2(b) as the film thickness increases the relative weight of $\mathrm{Ni}^{+3}$ ions progressively increases as well, causing the relative intensity of the peak near $870 \mathrm{eV}$ to decrease; the absorption spectrum for the $15 \mathrm{uc}$ film is characteristic of $\mathrm{Ni}^{3+}$ ions as confirmed by comparison to the reference $\mathrm{LaNi}(3+) \mathrm{O}_{3}$ absorption. To verify if the observed $\mathrm{Ni}^{2+}$ state is specific to the interface with polar discontinuity, we repeated the same XAS measurement on an identical 5 uc LNO film grown on LAO (111) (i.e. no polar jump at the interface). As shown in Fig. 2(b), in this case the $870 \mathrm{eV}$ peak characteristic of $\mathrm{Ni}^{2+}$ is absent and the resulting line shape and the absorption peak maximum is consistent with the bulk like $\mathrm{Ni}^{3+}$ charge state. This spectroscopic result is in good agreement with our diffraction data and lends strong support to the notion that the polarity mismatch at the LNO-STO interface stabilizes the $\mathrm{La}_{2} \mathrm{Ni}_{2}^{2+} \mathrm{O}_{5}$ phase in the ultra thin limit.

The presence of oxygen vacancies not only alters local charge states of nickel but also implies a difference in hybridization of the $\mathrm{Ni}-\mathrm{O}$ bond. The effect of oxygen vacancies was investigated by performing absorption measurements on the $\mathrm{O} K$-edge and monitoring the pre-edge region around $529 \mathrm{eV}$. This pre-edge intensity originates from a $3 d^{8} \underline{L} \rightarrow \underline{c} 3 d^{8}$ transition $^{43}$, where $\pm \underline{L}$ and $\underline{c}$ denote a ligand hole and an $\mathrm{O} 1 s$ core hole, respectively. In the past, the reduction of the $\mathrm{O} \mathrm{K}$ pre-edge intensity was used to monitor the oxygen vacancy formation in purposely reduced bulk compounds of $\mathrm{LaNiO}_{3}-\delta^{43}$. As shown in Fig. 2(c), the intensity of the pre-peak around $528.4 \mathrm{eV}$ for the $15 \mathrm{uc}$ film is very similar to that of the stoichiometric $\mathrm{LaNiO}_{3}$ and as the film thickness falls below 6 uc the pre-peak intensity disappears, implying a dramatic reduction in the degree of $\mathrm{Ni}-\mathrm{O}$ hybridization. This observation is in excellent conformance with the $\mathrm{Ni}^{2+}$ charge state deduced from the $\mathrm{Ni} L$-edge measurement.

Next we turn our attention to the question of how the electrical properties of ultra-thin LNO films are affected by the polar-misfit at the heterointerface. Fig. 3(a) shows temperature-dependent resistivities for the series of samples. As clearly seen, 15 uc and 10 uc films show bulk-like metallic behavior with a weak upturn at low temperature (inset of Fig. 3(a)). As the film thickness decreases, however, the resistivity increases and the $8 \mathrm{uc}$ film remains metallic only down to $180 \mathrm{~K}$; below this temperature the sample resistivity shows a semiconducting behavior. Consistent with the diffraction/absorption data discussed above, once the film thickness cell approaches $6 \mathrm{uc}$, the sample exhibits insulating (semiconducting) behavior starting at room temperature; at the critical thickness of 5 uc the film becomes highly insulating ( 2 orders of magnitude higher than the 6 uc film), 

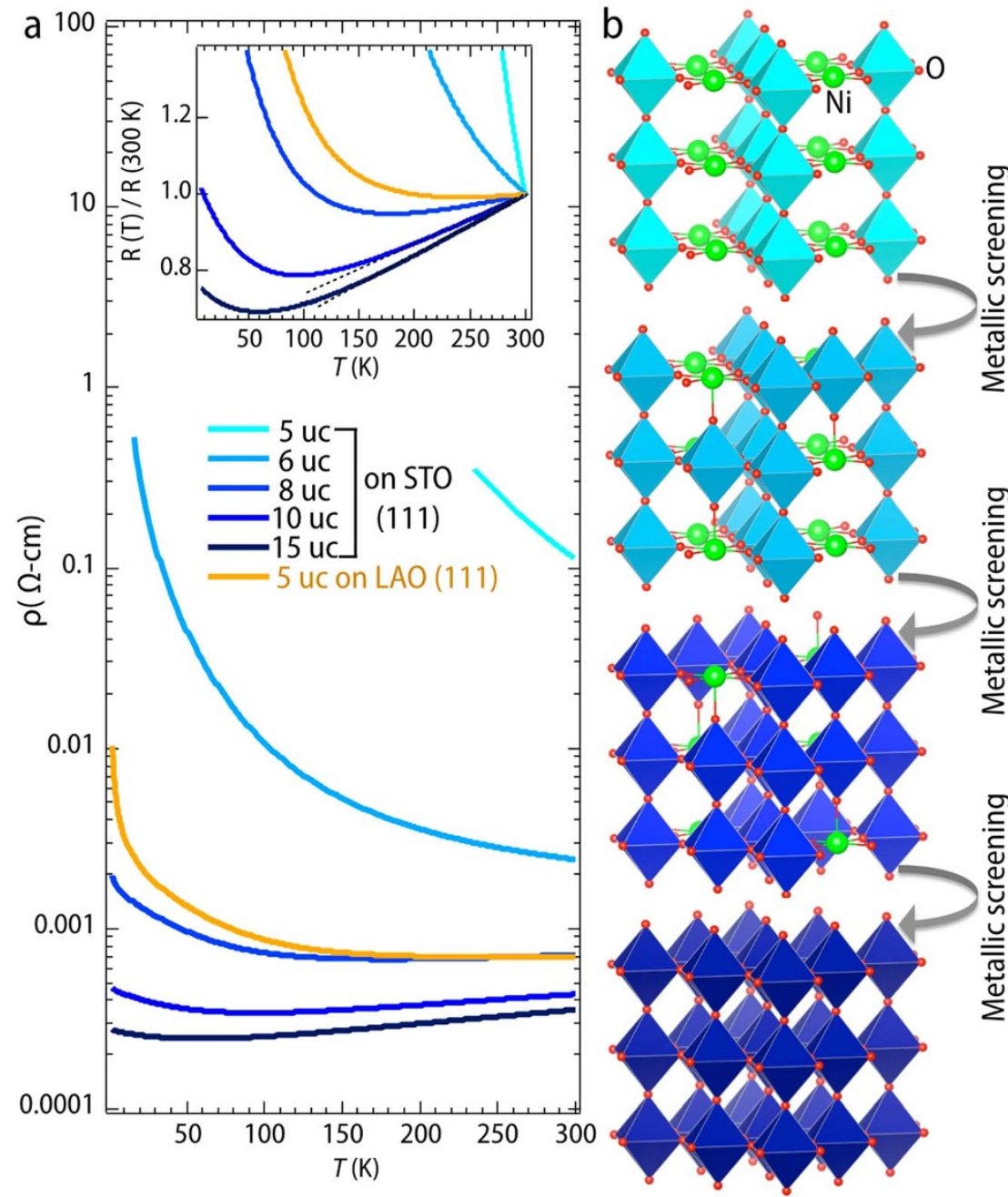

Figure $3 \mid$ Transport properties. (a) Resistivity as a function of temperature for LNO films on STO (111) with different thicknesses. Inset shows the variation of scaled resistivity. The dotted lines for 15 and $10 \mathrm{uc} \mathrm{film}$ indicate linear variation of $\rho$ with $T$ in metallic region. The enhancement of electrical conductivity with the film thickness partially screens the interfacial field, resulting in a gradual compensation of oxygen vacancies in $\mathrm{La}_{2} \mathrm{Ni}_{2} \mathrm{O}_{5}$ phase (formed during the first 5 uc growth). Such evolution of the $\mathrm{La}_{2} \mathrm{Ni}_{2} \mathrm{O}_{5+\delta}$ structure at the film-substrate interface has been shown schematically in (b). The color of the $\mathrm{NiO}_{6}$ octahedra represents the sample thickness mentioned in (a) by the same color of the $\rho$ vs. $T$ graph.

thus signifying the development of a new electronic ground state of the material.

While this metal-to-insulator transition as a function of film thickness could be linked to the effect of reduced dimensionality ${ }^{28,32}$, the two orders of magnitude reduction in resistivity for the 5 uc LNO film grown on non-polar LAO (111) and the high temperature MIT at $250 \mathrm{~K}$ point to the additional effects responsible for such highly insulating behavior of the 5 uc LNO film grown on STO (111). Again, the presence of a highly insulating LNO225 phase ${ }^{44}$ can account for the strongly disparate behavior, thus corroborating the conclusions deduced from the X-ray diffraction and XAS data. The details of these transport behavior are given in the Supplemental section.

Next we discuss the movement of the additional XRD peak, marked by the black solid triangle in Fig. 2(a). In general, it is assumed that oxygen vacancies in the perovskite $A B \mathrm{O}_{3-\delta}$ lattice are randomly arranged and characterized by the very large distribution of the local electronic environment ${ }^{45}$. Based on this, the experimentally determined structure at any given temperature can be viewed as a spatial and time average of the different local structures. Among the different possible structures with the formulae of $A_{2} B_{2} \mathrm{O}_{5}, \mathrm{La}_{2} \mathrm{Ni}_{2} \mathrm{O}_{5}$ adopts a very specific structure with alternating $\mathrm{NiO}_{6}$ octrahedra and $\mathrm{NiO}_{4}$ planar unit in the $a b$ plane (see top panel of Fig. 3(b)) due to the energetically unfavorable tetrahedral coordination of $\mathrm{Ni}^{+2}$ ions ${ }^{45,46}$. At the same time, the overall enhancement of electrical conductivity with the increase in film thickness acts to reduce the polar catastrophe by partial metallic screening. Thus some of the oxygen vacancies in $\mathrm{La}_{2} \mathrm{Ni}_{2} \mathrm{O}_{5}$ phase (formed during the first 5 uc growth) can be further effectively compensated by forming the $\mathrm{La}_{2} \mathrm{Ni}_{2} \mathrm{O}_{5+\delta}$ phase during the post annealing process under high oxygen pressure as shown schematically in Fig. 3(b). This results in a shift of the XRD peak position towards stoichiometric $\mathrm{LaNiO}_{3}$. We can also conjecture that when the metallic screening is sufficiently strong, the oxygen deficiencies are almost entirely compensated for as confirmed by the absence of the additional XRD peak for the $15 \mathrm{uc}$ film (Fig. 2(a)).

In order to understand how the oxygen vacancies screen the dipole field at the heterointerface, we performed ab-initio calculations for STO/LNO slabs oriented along (111), with a 15-uc-thick STO substrate and LNO layers of two different thickness (3 uc and $9 \mathrm{uc}$ ). The results are shown in Fig. 4. The single oxygen vacancy formation energy $E_{\text {form }}$ is defined as $E_{\mathrm{vac}}-E_{\text {full }}+\mu_{\mathrm{O}}\left(T, p_{\mathrm{O} 2}\right)$, where $E_{\mathrm{vac}}$ and $E_{\text {full }}$ is the free energy of a given slab with and without an oxygen vacancy respectively. The oxygen chemical potential $\mu_{\mathrm{O}}\left(T, p_{\mathrm{O} 2}\right)$ depends on the temperature $(T)$ and oxygen pressure $\left(p_{\mathrm{O} 2}\right)$ during growth or post annealing and can be approximated as $E_{\mathrm{dis}} / 2^{47}$, where 
$E_{\text {dis }}=-9.51 \mathrm{eV}$ is the dissociation energy to break up a single $\mathrm{O}_{2}$ bond. Fig. 4 (b) shows the oxygen vacancy formation energy $\left(E_{\text {form }}\right)$ as a function of distance from the LNO/STO interface for 3 (blue) and 9 (red) LNO layers. As seen in Fig. 4(b) the formation energy is always smaller (and hence preferable) for the vacancy created over the LNO side (positive values along the horizontal axis) compared to that on the STO side (negative values on the horizontal axis) for both $15 \mathrm{STO} / 3 \mathrm{LNO}$ and 15STO/9LNO systems. In addition, the calculation predicts a sharp decrease in $E_{\text {form }}$ upon approaching the surface monolayer of LNO (i.e. LNO/vacuum interface), and this result is found to be independent of the LNO slab thickness. We note, however, that first-principles calculations do not take into account the presence of an oxygen atmosphere during the growth and postannealing, which acts to compensate the vacancies at the surface monolayer ${ }^{18}$. As the oxygen ions are removed from a $\left[\mathrm{LaO}_{3}\right]^{-3}$ layer located deeper down from the $\mathrm{LNO} /$ vacuum interface, saturation of $E_{\text {form }}$ can be seen for the thicker LNO slab (9 LNO; this saturation of $E_{\text {form }}$ is emphasized by the yellow area in Fig. $4(\mathrm{~b})$ ). The shortest slab (3LNO) does not display such saturation due to the presence of the STO interface, where the formation energy continues to increase.
Comparing the two curves in Fig. 4(b), it can be easily seen that deep into the STO substrate $E_{\text {form }}$ takes the same value for both of the slabs considered regardless of LNO thickness (as indicated by the '*' mark in Fig. 4(b)), the formation of oxygen vacancies is always more favorable on LNO side for the 3LNO slab compared to 9 uc LNO. This corroborates the earlier discussed $\mathrm{Ni} L_{2}$ edge XAS results, showing the presence of larger number of $\mathrm{Ni}^{2+}$ ions in $5 \mathrm{uc}$ film compared to the thicker film.

The electrostatic potential energies shown for 15STO/9LNO and 15STO/3LNO slabs in Figs. 4 (a) and (c) respectively, further aid in understanding how the polar catastrophe can be avoided with oxygen vacancies. Those potentials are shown in black for systems without oxygen vacancies, and in green for slabs with a single oxygen vacancy at locations highlighted by vertical arrows. In addition, the mean potential energy, averaged over the length of a unit cell and displayed in red (blue) for slabs without oxygen vacancies (with a single oxygen vacancy), allows the reader to better view the potential profile across these slabs. The important observation is that the mean potential energy has a slower gradient and hence a smaller electric field (proportional to the gradient of potential) across the interface

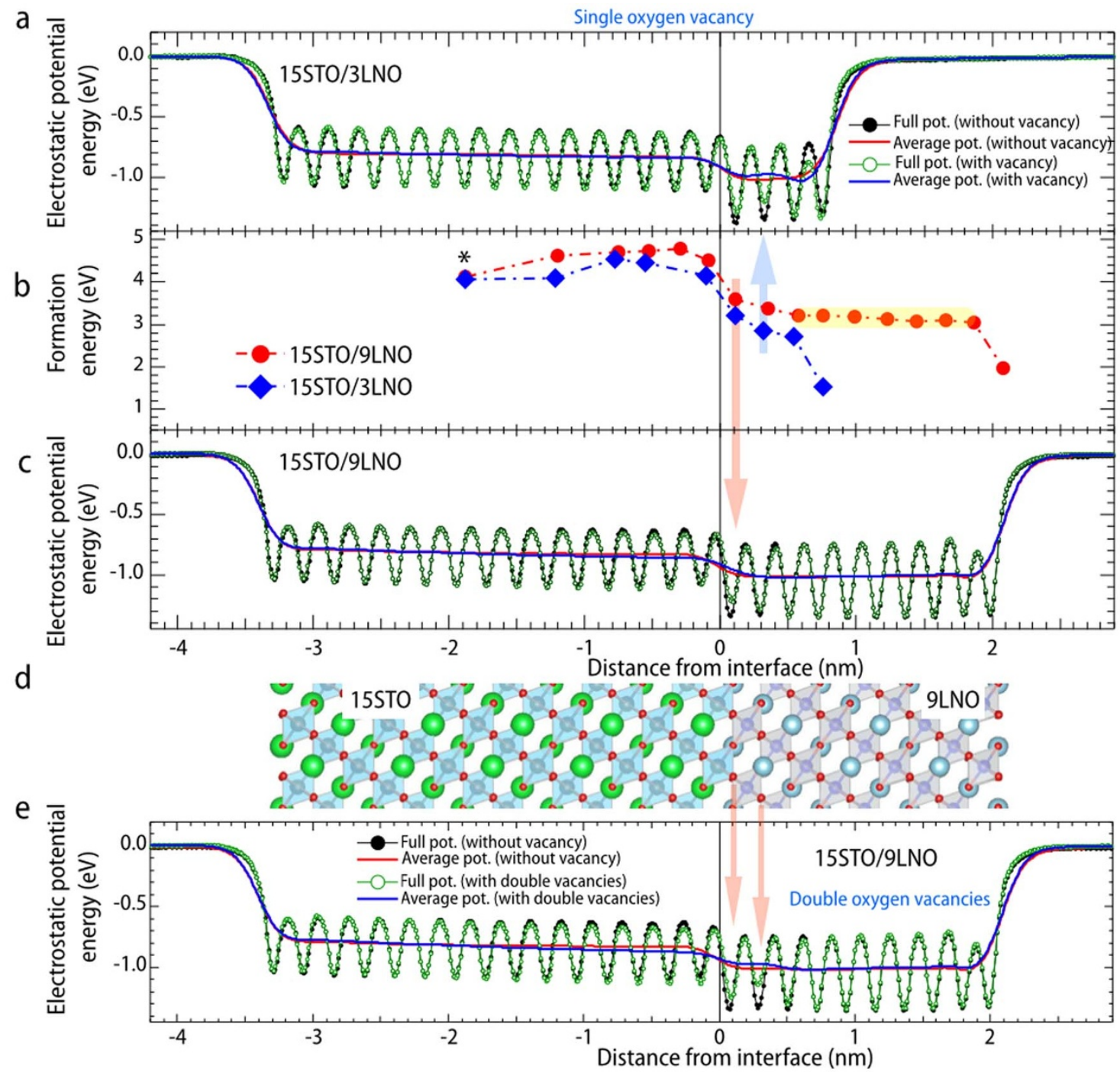

Figure $4 \mid$ Potential energy and oxygen-vacancy formation energy from first principles. (a and c) The electrostatic potential for 15STO/9LNO and 15STO/3LNO slabs with and without oxygen vacancy (the location of the vacancy has been shown by vertical arrow for both cases). (b) Oxygen-vacancy formation energy along the LNO/STO slab. Each of the data point corresponds to a calculation with a single vacancy at that location. (d) 15STO/9LNO slab used in calculations. (e) The electrostatic potential for 15STO/9LNO with two oxygen vacancies (positions of the vacancies are indicated by arrow in $(\mathrm{d}))$. 
for systems with oxygen vacancies formed at the vicinity of the heterointerface (blue trendlines) as compared to the system with no vacancies (red trendlines).

We have also explored the effects of oxygen di-vacancy on the potential energy profile across the STO/LNO system. Given a $15 \mathrm{STO} / 9 \mathrm{LNO}$ system with a vacancy close to the heterointerface, the calculation reveals that the second oxygen vacancy has its lowest formation energy when the second oxygen is removed from the same initial $\mathrm{NiO}_{6}$ octahedra but farthest away from the first vacancy site (shown by vertical arrows in Fig. 4(e)), thus forming a square coordinated by oxygen Ni unit characteristic of the LNO225 phase. As immediately seen in Fig. 4(e), the creation of this $2^{\text {nd }}$ vacancy results in an even smother slope of the average electrostatic potential (compare the smoother blue trend line vs. the red one). This result thus affirms a remarkably efficient suppression of the polar mismatch across the heterointerface and provides further microscopic insight into the role of oxygen vacancies as an efficient route for reducing, and even almost completely eliminating the polar mismatch at the oxides heterointerface.

To summarize, a series of epitaxial ultra-thin $\mathrm{LaNiO}_{3}$ films of different thicknesses (ranging from 5-15 unit cell) have been grown on polar STO (111) and non-polar LAO (111) substrates to investigate the effect of polar mismatch at the perovskite interface. A combination of in - situ RHEED imaging recorded during the growth of each consecutive unit cell, X-ray diffraction, transport and synchrotron based resonant $\mathrm{X}$-ray spectroscopy at $\mathrm{Ni}$ and $\mathrm{O}$ edges reveal the formation of polarity stabilized chemical phase $\mathrm{La}_{2} \mathrm{Ni}_{2} \mathrm{O}_{5}\left(\mathrm{Ni}^{2+}\right)$ for a few unit-cell thick films. A gradual increase of metallicity with increasing thickness serves as an additional mechanism to screen the diverging potential, leading to a sharp decrease of oxygen vacancies for thicker films and restoring nominal $3+$ charge state of $\mathrm{Ni}$. The combined experimental and $a b$-initio results demonstrate how the polarity at the oxide interface can be effectively controlled by stabilizing another structural and electronic phase formed within the first few monolayers. These findings should be taken into consideration in addition to electronic reconstruction and quantum confinement when describing the properties of ultra-thin films of complex oxides with polar discontinuity.

\section{Methods}

Experimental techniques. $\mathrm{LaNiO}_{3}$ thin films with different thicknesses were grown on high-quality STO (111) and LAO (111) substrates (Crystec, Germany) by pulsed laser interval deposition ${ }^{27,38,40}$ (laser frequency: $18 \mathrm{~Hz}$ ). In order to avoid additional surface defects on substrate, formed by the chemical treatment for achieving single termination $^{48}$, as received substrates were used. 50 mTorr partial pressure of oxygen was maintained during the growth and all of the grown samples were subsequently post annealed in-situ for $30 \mathrm{~min}$ in 1 atm of ultra pure oxygen at growth temperature $\left(670^{\circ} \mathrm{C}\right)$, which was found to be essential to maintain correct oxygen stoichiometry for the (001) oriented LNO/LAO heterostructures ${ }^{27}$. The films were characterized exsitu by laboratory-based XRD (Panalytical Xpert Pro MRD [Panalytical, Almelo]). Ni $L_{3,2}$ edge and $\mathrm{O} K$ edge XAS spectra were taken at room temperature at the 4-ID-C beam line of the Advanced Photon Source at Argonne National Laboratory. Electrical d.c. transport characterization was performed on a commercial physical properties measurement system (PPMS) with van der Paw geometry.

Theoretical methods. LDA calculations on $15 \mathrm{uc}$ STO/n-uc LNO multilayer slabs oriented along the (111) direction were carried out with the Vienna ab initio simulation package (VASP) ${ }^{49}$ using projector-augmented waves ${ }^{50,51}$. Total energies have been calculated for the stoichiometric structure with a $6 \times 6 \times 1 \mathrm{k}$-point sampling, with full structural relaxation and dipole corrections. The slabs have a $20 \AA-25 \AA$ vacuum in both sides to minimize artificial interactions among the periodic images. In computing oxygen vacancies, total electronic energies were calculated by removing one oxygen (for each calculation) from different layers with full structural atomic relaxation and fixed lattice vectors.

1. Chakhalian, J., Millis, A. J. \& Rondinelli, J. Whither the oxide interface. Nature Mater. 11, 92-94 (2012).

2. Hwang, H. Y. et al. Emergent phenomena at oxide interfaces. Nature Mater. 11, 103-113 (2012).

3. Yu, P., Chu, Y.-H. \& Ramesh, R. Oxide interfaces: pathways to novel phenomena. Mater. Today 15, 320-327 (2012).
4. Bibes, M., Villegas Javier, E. \& Barthélémy, A. Ultrathin oxide films and interfaces for electronics and spintronics. Advances In Physics 60, 5-84 (2010).

5. Ohtomo, A. \& Hwang, H. Y. A high-mobility electron gas at the $\mathrm{LaAlO}_{3} / \mathrm{SrTiO}_{3}$ heterointerface. Nature 427, 423-426 (2004).

6. Reyren, N. et al. Superconducting Interfaces Between Insulating Oxides. Science 317, 1196-1199 (2007).

7. Nakagawa, N., Hwang, H. Y. \& Muller, D. A. Why some interfaces cannot be sharp. Nature Mater. 5, 204-209 (2006).

8. Savoia, A. et al. Polar catastrophe and electronic reconstructions at the $\mathrm{LaAlO}_{3} /$ $\mathrm{SrTiO}_{3}$ interface: Evidence from optical second harmonic generation. Phys. Rev. B 80, 075110 (2009).

9. Sing, M. et al. Profiling the Interface Electron Gas of $\mathrm{LaAlO}_{3} / \mathrm{SrTiO}_{3}$ Heterostructures with Hard X-Ray Photoelectron Spectroscopy. Phys. Rev. Lett. 102, 176805 (2009).

10. Salluzzo, M. et al. Structural and Electronic Reconstructions at the $\mathrm{LaAlO}_{3} / \mathrm{SrTiO}_{3}$ Interface. Adv. Mater. 25, 2333 (2013).

11. Herranz, G. et al. High Mobility in $\mathrm{LaAlO}_{3} / \mathrm{SrTiO}_{3}$ Heterostructures: Origin, Dimensionality, and Perspectives. Phys. Rev. Lett. 98, 216803 (2007).

12. Park, J. et al. Oxygen-Vacancy-Induced Orbital Reconstruction of Ti Ions at the Interface of $\mathrm{LaAlO}_{3} / \mathrm{SrTiO}_{3}$ Heterostructures: A Resonant Soft-X-Ray Scattering Study. Phys Rev. Lett. 110, 017401 (2013).

13. Lin, W.-N. et al. Electrostatic Modulation of $\mathrm{LaAlO}_{3} / \mathrm{SrTiO}_{3}$ Interface Transport in an Electric Double-Layer Transistor. Adv. Mater. Interfaces 1, 1300001 (2014).

14. Hotta, Y., Susaki, T. \& Hwang, H. Y. Polar Discontinuity Doping of the $\mathrm{LaVO}_{3} /$ $\mathrm{SrTiO}_{3}$ Interface. Phys. Rev. Lett. 99, 236805 (2007).

15. Takizawa, M. et al. Spectroscopic evidence of competing interactions in polar multilayers $\mathrm{LaAlO}_{3} / \mathrm{LaVO}_{3} / \mathrm{LaAlO}_{3}$. Phys. Rev. Lett. 102, 236401 (2009).

16. Chambers, S. A. et al. Band allignment, built-In Potential, and the absence of conductivity at the $\mathrm{LaCrO}_{3} / \mathrm{SrTiO}_{3}$ (001) heterojunction. Phys. Rev. Lett. 107, 206802 (2011).

17. Akbashev, A. R. et al. Reconstruction of the polar interface between hexagonal $\mathrm{LuFeO}_{3}$ and intergrown $\mathrm{Fe}_{3} \mathrm{O}_{4}$ nanolayers. Sci. Rep. 2, 672 (2012).

18. Zhong, Z., Xu, P. X. \& Kelly, P. J. Polarity-induced oxygen vacancies at $\mathrm{LaAlO}_{3} /$ $\mathrm{SrTiO}_{3}$ interfaces. Phys. Rev. B 82, 165127 (2010).

19. Rüegg, A. \& Fiete, G. A. Topological insulators from complex orbital order in transition-metal oxides heterostructures. Phys. Rev. B 84, 201103(R) (2011).

20. Yang, K.-Y. et al. Possible interaction-driven topological phases in (111) bilayers of $\mathrm{LaNiO}_{3}$. Phys. Rev. B 84, 201104(R) (2011).

21. Wang, F. \& Ran, Y. Nearly at band with Chern number $C=2$ on the dice lattice. Phys. Rev. B 84, 241103(R) (2011).

22. Xiao, D. et al. Interface engineering of quantum Hall effects in digital transition metal oxide heterostructures. Nat. Commun. 2, 596 (2011).

23. Rüegg, A. et al. Electronic structure of $\left(\mathrm{LaNiO}_{3}\right)_{2} /\left(\mathrm{LaAlO}_{3}\right)_{N}$ heterostructures grown along 111. Phys. Rev. B 85, 245131 (2012).

24. Okamoto, S. Doped Mott Insulators in (111) Bilayers of Perovskite TransitionMetal Oxides with a Strong Spin-Orbit Coupling. Phys. Rev. Lett. 110, 066403 (2013).

25. Rüegg, A. et al. Lattice distortion effects on topological phases in $\left.\mathrm{LaNiO}_{3}\right)_{2} /$ $\left(\mathrm{LaAlO}_{3}\right)_{N}$ heterostructures grown along the 111. direction. Phys. Rev. B 88, 115146 (2013).

26. Blok, J. L. et al. Epitaxial oxide growth on polar (111) surfaces. Appl. Phys. Lett. 99, 151917 (2011).

27. Liu, J. et al. Effect of polar discontinuity on the growth of $\mathrm{LaNiO}_{3} / \mathrm{LaAlO}_{3}$ superlattices. Appl. Phys. Lett. 96, 133111 (2010).

28. Liu, J. et al. Quantum confinement of Mott electrons in ultrathin $\mathrm{LaNiO}_{3} / \mathrm{LaAlO}_{3}$ superlattices. Phys. Rev. B 83, 161102(R) (2011).

29. Freeland, J. W. et al. Orbital control in strained ultra-thin $\mathrm{LaNiO}_{3} / \mathrm{LaAlO}_{3}$ superlattices. Euro Phys. Lett. 96, 57004 (2011).

30. Boris, A. V. et al. Dimensionality Control of Electronic Phase Transitions in Nickel-Oxide Superlattices. Science 332, 937-940 (2011).

31. Benckiser, E. et al. Orbital reectometry of oxide heterostructures. Nature Mater. 10, 189-193 (2011)

32. Scherwitzl, R. et al. Metal-insulator transition in ultrathin $\mathrm{LaNiO}_{3}$ films. Phys. Rev. Lett. 106, 246403 (2011).

33. Chakhalian, J. et al. Asymmetric Orbital-Lattice Interactions in Ultrathin Correlated Oxide Films. Phys. Rev. Lett. 107, 116805 (2011).

34. Chaloupka, J. \& Khaliullin, G. Orbital Order and Possible Superconductivity in $\mathrm{LaNiO}_{3} / \mathrm{LaMO}_{3}$ Superlattices. Phys. Rev. Lett. 100, 016404 (2008).

35. Hansmann, P. et al. Turning a Nickelate Fermi Surface into a Cupratelike One through Heterostructuring. Phys. Rev. Lett. 103, 016401 (2009).

36. Rödel, T. C. et al. Orientational Tuning of the Fermi Sea of Confined Electrons at the $\mathrm{SrTiO}_{3}$ (110) and (111) Surfaces. Phys. Rev. Appl. 1, 051002 (2014).

37. Wang, S. C. P. et al. Deposition of in-plane textured $\mathrm{MgO}$ on amorphous $\mathrm{Si}_{3} \mathrm{~N}_{4}$ substrates by ion-beam-assisted deposition and comparisons with ion-beamassisted deposited yttria-stabilized-zirconia. Appl. Phys. Lett. 71, 2955 (1997)

38. Kareev, M. et al. Sub-monolayer nucleation and growth of complex oxides at high supersaturation and rapid flux modulation. J. Appl. Phys. 109, 114303 (2011).

39. Middey, S. et al. Epitaxial stabilization of ultra thin films of electron doped manganites. Appl. Phys. Lett. 104, 202409 (2014).

40. Middey, S. et al. Epitaxial growth of (111)-oriented $\mathrm{LaAlO}_{3} / \mathrm{LaNiO}_{3}$ ultra-thin superlattices. Appl. Phys. Lett. 101, 261602 (2012). 
41. Alonso, J. A. \& Martinezlope, M. J. Preparation and crystal-structure of the defficient perovskite $\mathrm{LaNiO}_{2.5}$, solved from neutron diffraction data. J. Chem. Soc., Dalton Trans. 1995, 2819-2824 (1995).

42. Crespin, M., Levitz, P. \& Gatineau, L. Reduced forms of $\mathrm{LaNiO}_{3}$ perovskite 1 . evidence for new phases $-\mathrm{La}_{2} \mathrm{Ni}_{2} \mathrm{O}_{5}$ and $\mathrm{LaNiO}_{2}$. J. Chem. Soc. Faraday Trans. 79 , 1181 (1983).

43. Abbate, M. et al. Electronic structure and metal-insulator transition in $\mathrm{LaNiO}_{3} \delta$ Phys. Rev. B 65, 155101 (2002).

44. Sánchez, R. D. et al. Metal-insulator transition in oxygen-deficient $\mathrm{LaNiO}_{3-x}$ perovskites. Phys. Rev. B 54, 16574 (1996).

45. Stølen, S., Bakken, E. \& Mohn, C. E. Oxygen-deficient perovskites: linking structure, energetics and ion-transport. Phys. Chem. Chem. Phys. 8, 429 (2006).

46. Stølen, S., Mohn, C. E., Ravundran, P. \& Allan, N. L. Topography of the Potential Energy Hypersurface and Criteria for Fast-Ion Conduction in Perovskite-Related $\mathrm{A}_{2} \mathrm{~B}_{2} \mathrm{O}_{5}$ Oxides. J. Phys. Chem. B 109, 13262 (2005).

47. Posadas, A. B. et al. Oxygen vacancy-mediated room-temperature ferromagnetism in insulating cobalt-substituted $\mathrm{SrTiO}_{3}$ epitaxially integrated with silicon. Phys. Rev. B 87, 144422 (2013).

48. Zhang, J. et al. Depth-resolved subsurface defects in chemically etched $\mathrm{SrTiO}_{3}$. Appl. Phys. Lett. 94, 092904 (2009).

49. Kresse, G. \& Furthmüller, J. Efficient iterative schemes for ab initio total-energy calculations using a plane-wave basis set. Phys. Rev. B 54, 11169 (1996).

50. Blöchl, P. E. Projector augmented-wave method. Phys. Rev. B 50, 17953 (1994).

51. Kresse, G. \& Joubert, D. From ultrasoft pseudopotentials to the projector augmented-wave method. Phys. Rev. B 59, 1758 (1999).

\section{Acknowledgments}

J. C. was supported by DOD-ARO under Grant No. 0402-17291 and DOE under Grant no 0402 81814-21-0000. Work at the Advanced Photon Source, Argonne was supported by the
U.S. Department of Energy, Office of Science under Grant No. DEAC02-06CH11357. S. M. thanks M. Hawkridge for the help in XRD measurements and S. B.-L. thanks L. Bellaiche and $\mathrm{H}$. Fu for discussions and funding from Arkansas Biosciences Institute. Calculations were carried out at TACC (Stampede, Grant XSEDE TG-PHY090002) and Razor (Arkansas).

\section{Author contributions}

S.M. and M.K. prepared the sample. S.M. measured diffraction, transport property and analyzed all experimental data. S.M., D.M., X.L., Y.C. and J.W.F. acquired XAS data. P.R and S.B.-L. carried out theoretical calculations. All authors contributed to the data interpretations. S.M., D.M., S.B.-L. and J.C. wrote the manuscript with input from all authors. J.C. supervised the work.

\section{Additional information}

Supplementary information accompanies this paper at http://www.nature.com/ scientificreports

Competing financial interests: The authors declare no competing financial interests. How to cite this article: Middey, S. et al. Polarity compensation in ultra-thin films of complex oxides: The case of a perovskite nickelate. Sci. Rep. 4, 6819; DOI:10.1038/ srep06819 (2014)

This work is licensed under a Creative Commons Attribution-NonCommercialNoDerivs 4.0 International License. The images or other third party material in this article are included in the article's Creative Commons license, unless indicated otherwise in the credit line; if the material is not included under the Creative Commons license, users will need to obtain permission from the license holder in order to reproduce the material. To view a copy of this license, visit http:// creativecommons.org/licenses/by-nc-nd/4.0/ 